\title{
Symbiosis Evolution of Science Communication Ecosystem Based on Social Media: A Lotka-Volterra Model-Based Simulation
}

\author{
Ming Xia $\mathbb{D}^{1},{ }^{1}$ Xiangwu He $\mathbb{D}^{2},{ }^{2}$ and Yubin Zhou $\mathbb{D}^{1}$ \\ ${ }^{1}$ School of Economics and Management, Tongji University, Shanghai 200092, China \\ ${ }^{2}$ Business School, Jiaxing University, Jiaxing 314001, China \\ Correspondence should be addressed to Yubin Zhou; dazhou@tongji.edu.cn
}

Received 15 December 2020; Revised 27 February 2021; Accepted 1 April 2021; Published 15 April 2021

Academic Editor: Jiaojiao Jiang

Copyright (c) 2021 Ming Xia et al. This is an open access article distributed under the Creative Commons Attribution License, which permits unrestricted use, distribution, and reproduction in any medium, provided the original work is properly cited.

Social media has become an important way for science communication. Some scholars have examined how to help scientists engage with social media from operational training, policy guidance, and social media services improving. The main contribution of this study is to construct a symbiosis evolution model of science communication ecosystem (SCE) between scientists and social media platforms based on the symbiosis theory and the Lotka-Volterra model to discuss the evolution of their symbiotic patterns and population size under different symbiosis coefficients. The results indicate that (1) the size of the symbiosis coefficients determines the equilibrium outcome of the symbiosis evolution of scientists and social media platforms; (2) scientists and social media platforms can promote each other's population size under the mutualism pattern, which can achieve sustainable science communication; (3) " $1+1>2$ " effect can only be achieved under the symmetric mutualism pattern and the growth of scientists and social media platforms is more stable and sufficient than that of other patterns. The findings will provide additional perspectives for promoting the sustainable development of science communication based on social media.

\section{Introduction}

The communication between scientists and the public is changing as we enter the information age. It is a fact of mass communication effect research that exposure is a necessary condition for media effect and that communication activities often fail due to insufficient exposure [1]. The rapid development of new media, such as social media, has brought tremendous changes to the technological environment of science communication [2]. It has become one of the most influential factors in science communication, and the study of media as an environmental factor has become a research hotspot [3].

Social media has dramatically changed the ecological environment of science communication. The traditional form of media essentially disseminates information from a single point to the target audience [4]. The emergence of social media has created a domain-based electronic community that facilitates information interaction $[5,6]$. In addition to changing the way information is disseminated, shortening the temporal and spatial distance between the public and the scientific community, and creating conditions for public participation in scientific research, changes in the technological environment have more importantly enriched the vector of dissemination of scientific information. Since the publication of Robert Hook's micrographs, images have been placed at the center of the modern science communication process [7]. In the era of digital communication, technological advances have enriched the transmission medium of science and technology information. The development of video images, virtual reality, augmented reality, and mixed reality technology has created a visual information-intensive environment for science communication and greatly expanded the dimension of interaction between experts and the public [8].

In recent years, recognizing the enormous potential of the changing technological environment at the level of knowledge exchange and knowledge diffusion, the academic community has begun to experiment with the use of social media as a way to communicate their academic ideas to the 
public [9]. Through the use of social media in the process of academic research, social media has become structurally embedded in the scholarly communication system [10]. Scholars have confirmed the possibility of using social media for scientific research [11, 12]. Previous research has established that researchers can build connections and conduct academic exchanges through social media and obtain new research ideas from them, and there is evidence of a positive trend in this kind of use [13-15]. However, statistics show that most scientists have yet to use social media such as Facebook, Twitter, WeChat, and Weibo in their professional or academic work [16]. Collins et al. (2012) surveyed a large number of scholars from the US and UK on the use of social media and found that $3 \%$ of them are highly active on Twitter [17]. These data are far from the $23 \%$ of American adult users owned by Twitter and $71 \%$ of American adult users owned by Facebook. This gap also shows that the habit of using social media in academic workplaces has not yet been developed [18].

Therefore, what prevents scientists from using social media to engage in science communication? Most scholars believe that scientists are supposed to be dedicated to their research, not to the promotion of their findings on social media, and that the dissemination of science through the media results in the undermining of scientists' authority [19]. "Sagan Effect" has been used to describe scientists who "risk the contempt of serious scientists by becoming science communicators." The bias can be roughly summed up as scientists who are diligent in science communication are second-rate scientists [20]. After the gradual development of science communication, more scientists have emerged to participate in science communication, but the "Sagan Effect" still influences scientists' choices. Besides, it has been shown that some scientists are fearful of using social media due to a lack of knowledge about the usage of social media, privacy, and other considerations, which leads to knowledge barriers at the level of ability to use social media [21]. "Lack of time" is another important influencing factor, time wastage has been identified as an important barrier to scientists' outreach [22], in the field of science communication, the use of social media is considered a waste of time by some researchers $[23,24]$, as well as the lack of general clarity on the benefits of participating in social media, and the fact that social media themselves are at odds with scientific rigor make social media unattractive to scientists [25].

Further, how to help scientists use social media? Collins et al. (2016) advocate guiding scientists to engage with social media by conducting professional development training workshops or a more explicit departmental social media use policy [26]. Training usually includes helping scientists find and clarify their message, learning to work with media professionals, and preparing for the use of social media [27-29]. The science communication training program has developed rapidly in recent years; it emphasizes technical communication skills and guides scientists to find their communication opportunities. Exemplars of programs include Alan Alda Center for Communicating Science, the Center for Public Engagement with Science and Technology at the American Association for the Advancement of
Science, COMPASS, and the Portal to the Public Network $[30,31]$. From the perspective of social media platforms, some scholars have studied the service upgrades made by social media platforms to attract scientists and other content creators [32]. To be an attractive platform for users (especially content creators), social media offers a variety of features, such as allowing users to create their channels, postcontent that can be immediately shared with a wide audience, choose to become friends with others, or subscribe to other channels, and then comment on or choose the content what you like [33]. Features provide an incentive for content creators, including scientists. Also, content creators can share revenue generated by user donations through the platform, which supports social media to ensure its innovation and long-term viability [34]. The scale effect of social media can attract more content creators to participate in science communication [35]. Similarly, as a major force of science communication, the increase in the number of groups of scientists is indicative of a strong user demand that can lead to more niche social media-based science communication platforms $[36,37]$. There is a symbiosis relationship between scientists and social media platforms, and the population numbers between scientists and social media platforms influence each other.

In summary, scholars have researched the current situation of scientists' participation in social media, the reasons for the lack of participation vitality, and the measures that should be taken and have achieved relatively rich results. A review of the literature reveals that existing research mainly focuses on the individual level of scientists and social media platforms. Scholars hope to achieve compatibility between scientists and social media on individual changes, thereby enhancing the vitality of scientists to participate in social media. From an ecological point of view, scientists and social media platforms, as two symbiotic units in the science communication ecosystem (SCE), are symbiosis relationship, and a change in the population size of one will have an impact on the population size of the other inevitably. In this study, we discuss the population symbiosis between scientists and social media platforms from an ecological perspective, establish a Lotka-Volterra population competition model between scientists and social media platforms, and investigate the symbiosis evolution between them with different symbiosis coefficients under a certain environmental carrying capacity, to complement the research about scientists' use of social media for sustainable science communication.

The remainder of this study is mainly divided into the following sections. Section 2 is preliminary. Section 3 introduces the symbiosis evolution model of the SCE based on the Lotka-Volterra model. Sections 4 and 5 are simulation analyses and conclusions, respectively.

\section{Preliminary}

2.1. Symbiosis Theory. The concept "symbiosis" is of Greek origin and was first introduced by the German mycologist Anto de Bary in 1879 to refer to the concept of different genera living together in an extended material relationship 
[38]. Symbiosis is a self-organizing phenomenon and the need for survival between living organisms is inevitably interdependent and interactive in some way, forming a symbiosis relationship of coexistence and synergistic evolution [39]. However, the concept of symbiosis has not yet been harmonized due to the influence of different disciplinary contexts. Symbiosis in the narrower sense is considered as a reciprocal, mutually beneficial cooperative relationship between species. The German scientist Buchner considered symbiosis to be an intimate, enduring union between two dissimilar organisms and tended to limit it to a mutually beneficial union [40]. Dale Weis defines symbiosis as a stable, enduring, and intimate combined relationship between several pairs of cooperators [41]. In a broad sense, symbiosis is the existence of power relationships between species such as metabolism and energy conversion. Margulis, an American biologist, pointed out from an ecological point of view that "symbiosis is the union of important parts of the life cycles of individuals of different species" [39]. Goff (1982) pointed out that symbiosis includes various degrees of parasitism, commensalism, and mutualism [38].

Based on the symbiosis theory, Yuan (1998) discussed the three elements: symbiotic units, symbiotic patterns, and symbiotic environment [42]. The symbiotic units are the basic units of energy production and exchange that constitute the symbiosis relationship, which is the basis for the existence of the symbiosis relationship; the symbiotic pattern, also known as the symbiosis relationship, is the form of interaction or combination of symbiotic units, which reflects not only the pattern of action between the symbiotic units but also the intensity of action, which reflects the exchange of material information between the symbiotic units, as well as the exchange of energy between the symbiotic units; the symbiotic environment refers to the exogenous conditions for the development of the existence of the symbiotic pattern, and the sum of all factors other than the symbiotic units constitute the symbiotic environment, which is the guarantee for the survival of the symbiotic units. Three elements are indispensable as shown in Figure 1.

2.2. Structure of SCE. SCE based on social media is a complex system composed of multiple individual users gathered on a certain type of social media platform and formed by the interconnection and influence of its external network environment and the real social environment. Similar to the natural ecosystem, the SCE has a continuous evolutionary process from creation, expansion, maintenance to decline. The structure is an important indicator of the balance and sustainability of an ecosystem $[43,44]$. The structure of the SCE refers to the combination of the relatively stable interaction patterns of various components and the internal manifestations of their temporal and spatial relationships. This study focuses on the analysis of the symbiosis relationship between scientists and social media platforms in terms of the ecological chain structure of the SCE and the flow of information resources.

The ecological chain structure of the ecosystem mainly refers to the network structure formed by the chain network between biological components [45]. Take natural ecosystems as an example. Although natural ecosystems vary greatly in appearance, structure, and feature, each natural ecosystem has three main elements, producers, consumers, and decomposers, and realizes the smooth flow of matter and energy through the above three main elements $[46,47]$. The SCE belongs to the category of the social ecosystem, but it still has the relevant elements of the natural ecosystem. These similarities are manifested in the similar ecological chain structure of the natural ecosystem and the science ecosystem, but they are still different, as shown in Figure 2.

The natural ecological chain is composed of producers, multilevel consumers, and decomposers. Producers and primary consumers, primary consumers, and secondary consumers form an ecological sequence through the preying relationship. Producers and consumers involved in each link of energy flow are decomposed and released into the environment again to form a closed loop of energy flow.

The difference between the science communication ecological chain and the natural ecological chain is mainly reflected in the changes in the relationship between producers and consumers. The relationship between producers and consumers in the natural ecological chain is predation. Energy flow is a one-way flow from producers to consumers [48]. However, there is no traditional "predation" relationship between scientists and the public in the science communication ecological chain, or the meaning of "predation" has been transformed into a two-way symbiosis relationship between scientists' access to public attention and the public's access to knowledge from scientists. Scientists and social media platforms, social media platforms, and the public form a symbiosis relationship through the flow of information, and because of the addition of a twoway adjustment mechanism with feedback, the way of information flow has also changed from one-way to two-way, and the positive direction of information flow represents the process of scientific communication, and reverse flow represents the process of information consultation and feedback.

\section{Methodology}

This section will introduce the background and development status of the Lotka-Volterra model at first and then build a model of the symbiosis evolution of SCE based on the Lotka-Volterra model considering the specific context of the issue. Finally, we will discuss the different symbiosis relationships under different symbiosis coefficients and the stable conditions at this time.

3.1. Lotka-Volterra Model. In this paper, the authors attempt to explore the symbiosis and evolution of scientists and social media platforms in the SCE with computer simulation methods. The SCE based on social media is an ecosystem composed of scientific communities, social media platforms, users, and so on. This study draws on the ecological evolution method to explore the number, structure, and overall evolution of its internal population. From an 


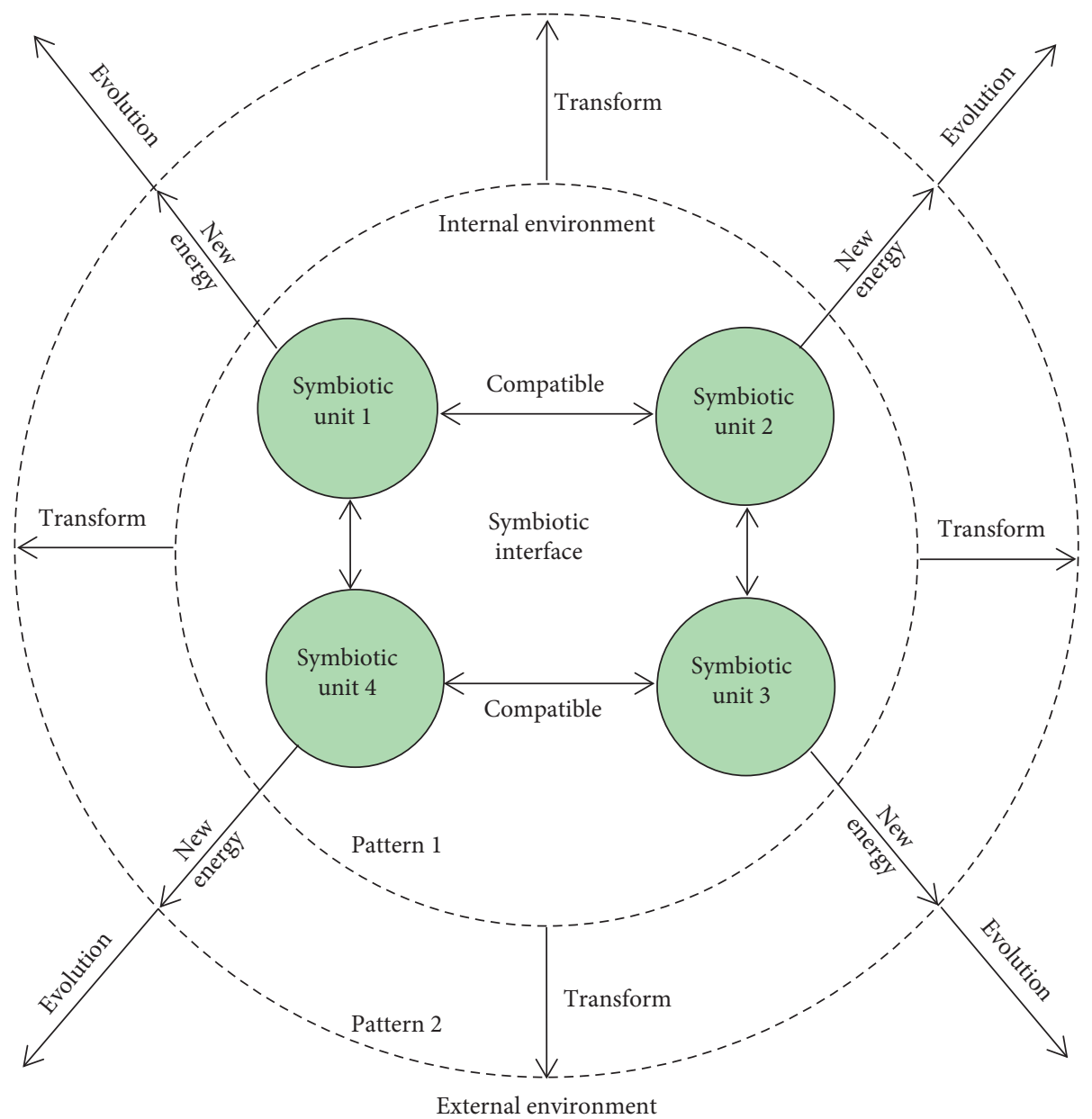

Figure 1: Symbiosis evolution of symbiotic units. The symbiotic units exchange energy and information through quality parameters on the symbiotic interface, and the new energy generated changes the symbiosis pattern and gradually realizes the symbiosis evolution.

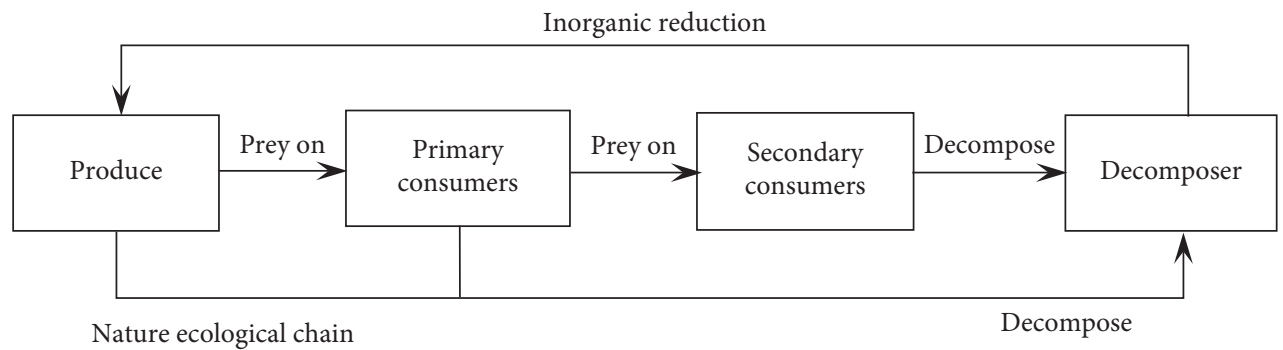

Nature ecological chain

Decompose

"Bidirectional predation relationship"

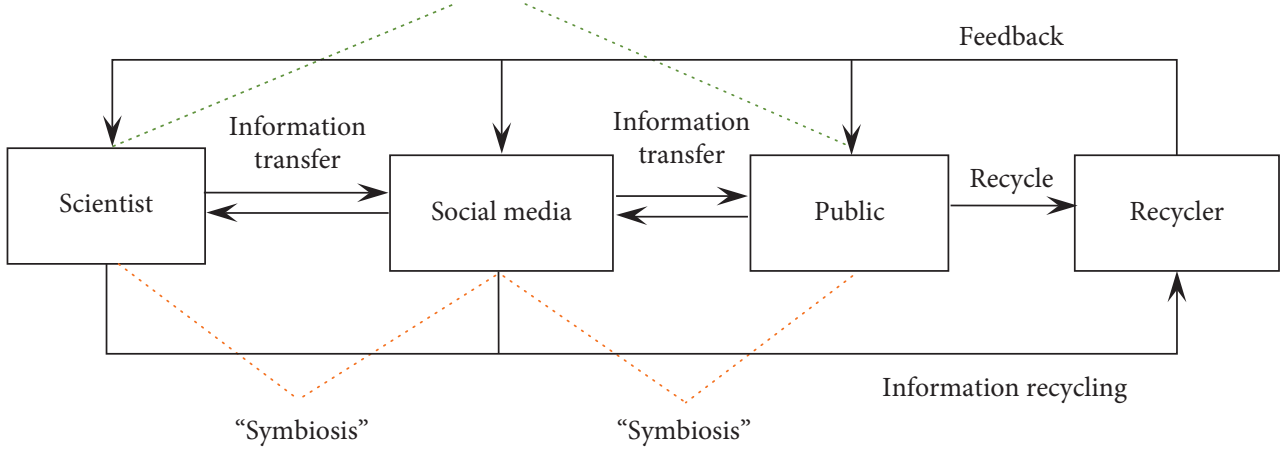

Science communication ecological chain

Figure 2: Comparison between nature ecological chain and science communication ecological chain. The direction of the arrow indicates the direction of information or energy flow, and the dashed line indicates the relationship between the two sides. 
ecological point of view, the growth and change law of biological populations is as follows: in a short period, generally follow the Malthus model growth law; that is, the population size grows exponentially; with the growth of time, the population size gradually increases, the influence of density constraints is increasing and showing the logistic growth law, and the growth rate of population size becomes slower and gradually reaches saturation. The differential equation expression is shown in model

$$
\frac{\mathrm{d} N(t)}{\mathrm{d} t}=\alpha N(t)\left(1-\frac{N(t)}{N^{*}}\right) .
$$

In model (1), suppose that there is one population in the ecosystem under a certain natural growth rate $\alpha$. The population size $N(t)$ will gradually change over time and reach the maximum population size $N^{*}$ in a specific environment eventually. The logistic model was first applied to the estimation of population, and then it was widely used in population ecology and sociology [49].

The Lotka-Volterra model is proposed by Lotka and Volterra based on a single species logistic model, which considers the dynamic growth of two or more populations in the ecosystem at the same time [50], as shown in model

$$
\frac{\mathrm{d} N_{1}(t)}{\mathrm{d} t}=\alpha_{1} N_{1}(t)\left(1-\frac{N_{1}(t)}{N_{1}^{*}}-\delta \frac{N_{2}(t)}{N_{2}^{*}}\right) .
$$

In model (2), suppose that there are two populations in the ecosystem under a certain natural growth rate $\alpha_{1}$. The population size of each species is affected not only by the size of its population but also by the size of another population. The population size $N_{1}(t)$ will gradually change over time and reach the maximum population size $N_{1}^{*}$ in a specific environment eventually.

Lotka-Volterra model has good data fitting and prediction performance and is widely used in research in various fields such as economics, business, urbanization, and sociology. In economic research, Bernardo and D'Alessandro (2016) studied a macroeconomic framework based on the Lotka-Volterra model to assess the social and economic consequences of investing in lowcarbon options [51]. In business research, Khan et al. (2018) studied the impact of low-cost airlines on Korean full-service companies and tourism based on the Lotka-Volterra model [52]. In the urbanization research, Assumma et al. (2019) discussed how to use the system dynamics model and the Lotka-Volterra model to assess regional elasticity [53]. In sociological research, Marasco and Romano (2018) used Lotka-Volterra to study the intergenerational conflicts in the American aging society from 1974 to 2014 [54]. Ditzen (2018) studied the multinational convergence of the Lotka-Volterra model [55]. Christodoulakis (2016) used the dynamic Lotka-Volterra model to quantitatively analyze the armed confrontation that occurred in Greece from 1946 to 1949 and explained the dynamics and costs of the conflict in the Greek Civil War [56].

\subsection{Analysis of the Symbiosis Evolution}

\subsubsection{Hypothesis}

Hypothesis 1. There is a certain number of scientists and social media platforms in the SCE. The population of scientists and social media platforms is affected by factors such as policy, economy, society, infrastructure, technology, scientific research environment, and science communication industry environment.

Hypothesis 2. Changes in the scale of scientists and social media platforms in the social media ecology reflect their respective growth conditions. The positive change in the population size indicates the use of various science resources in the SCE. The higher the degree, the greater the gains obtained during the evolution of the symbiosis relationship and the better the growth; the reverse change of the population size indicates that the population has a low degree of utilization of various scientific resources in the SCE in the context of science communication, and the symbiosis relationship is evolving. The income obtained is low, and the growth of the population is poor. When the utilization rate of a population for popular science resources is 0 , it indicates that the population is extinct.

Hypothesis 3. The growth process of the group of scientists and social media platforms obeys the logistic growth law. Due to the limitation of the capacity of the ecosystem, the population growth rate when each participant grows alone will gradually decrease as the population density increases.

Hypothesis 4. When the marginal output of the population of scientists or social media platforms equals the marginal input, the population growth rate tends to 0 , and the population reaches its maximum size.

3.2.2. Symbiosis Evolution Model. Assume that the two groups grow independently and do not affect each other. We suppose that the population numbers of scientists and social media platforms are $N_{1}$ and $N_{2}$ and the natural growth rates are $\alpha_{1}$ and $\alpha_{2}$, respectively. $N_{1}^{*}$ and $N_{2}^{*}$ are here used to refer to the maximum size of the two types of the population under the given resource elements. The dynamic evolution equations of the two types of symbiotic units in the SCE can be expressed as equation group

$$
\left\{\begin{array}{l}
\frac{\mathrm{d} N_{1}(t)}{\mathrm{d} t}=\alpha_{1} N_{1}(t)\left[1-\frac{N_{1}(t)}{N_{1}^{*}}\right], \\
N_{1}(0)=N_{10}, \\
\frac{\mathrm{d} N_{2}(t)}{\mathrm{d} t}=\alpha_{2} N_{2}(t)\left[1-\frac{N_{2}(t)}{N_{2}^{*}}\right], \\
N_{2}(0)=N_{20} .
\end{array}\right.
$$


In the equation group (3), the initial size of the population of scientists and social media organizations in social media networks is $N_{10}$ and $N_{20}$, respectively. $\alpha_{1} N_{1}(t)$ and $\alpha_{2} N_{2}(t)$ are used here to refer to reflect the number of scientists participating in social media and the development trend of the population of social media platforms; $1-\left(N_{1}(t) / N_{1}^{*}\right)$ and $1-\left(N_{2}(t) / N_{2}^{*}\right)$ are the logistic coefficient, which is used here to refer to the hindrance of the growth of their scale due to the consumption of limited resources by the two types of participants, respectively.

Considering the interaction between populations and according to the symbiosis theory, the two types of symbiotic units will undergo a transition from one symbiosis pattern to another during the development process, embodying different symbiosis effects and forming different symbiosis relationships [57]. When two types of symbiotic units interact in the SCE, the growth of each type of symbiotic unit is not only affected by its population size but also related to the population size of the other type of symbiotic units. According to the Lotka-Volterra model, the symbiosis coefficient $\delta$ is here introduced to reflect the size of the symbiosis effect. Based on this, the dynamic model of the interaction and evolution of the population of social media scientists and social media organizations can be expressed as equation group

$$
\left\{\begin{array}{l}
\frac{\mathrm{d} N_{1}(t)}{\mathrm{d} t}=\alpha_{1} N_{1}(t)\left[1-\frac{N_{1}(t)}{N_{1}^{*}}-\delta_{21} \frac{N_{2}(t)}{N_{2}^{*}}\right], \\
N_{1}\left(t_{0}\right)=N_{10}, \\
\frac{\mathrm{d} N_{2}(t)}{\mathrm{d} t}=\alpha_{2} N_{2}(t)\left[1-\frac{N_{2}(t)}{N_{2}^{*}}-\delta_{12} \frac{N_{1}(t)}{N_{1}^{*}}\right], \\
N_{2}\left(t_{0}\right)=N_{20} .
\end{array}\right.
$$

In equation group (4), $\delta_{12}$ and $\delta_{21}$ are symbiosis coefficient, which is used here to indicate the symbiosis effect of scientists participating in social media on the population size of social media organizations and social media organizations on the population size of scientists participating in social media. That is, the changes of the quality parameter of the other party caused by the interaction between the quality parameters $N_{1}(t)$ and $N_{2}(t), \quad \delta_{21}\left(N_{2}(t) / N_{2}^{*}\right)$ and $\delta_{12}\left(N_{1}(t) / N_{1}^{*}\right)$ indicate the degree of influence caused by both parties, and the values of $\delta_{12}$ and $\delta_{21}$ reflect the symbiotic pattern of the two types of symbiosis units in the SCE.

3.2.3. Model Analysis. According to the different value ranges of $\delta_{12}$ and $\delta_{21}$, the Lotka-Volterra model of the interaction of the two types of symbiotic units and their symbiosis relationship is discussed in Table 1 .

It can be seen that the result of the symbiosis evolution between the group of scientists and social media organizations in the SCE depends on the value of the symbiosis coefficient. To study the symbiosis evolution relationship between scientists and social media organizations, it is necessary to analyze the equilibrium point and stability conditions of the dynamic evolution equation and analyze the symbiosis evolution results of the two types of symbiotic units. When $\left(\mathrm{d} N_{1}(t) / \mathrm{d} t\right)=0$ and $\left(\mathrm{d} N_{2}(t) / \mathrm{d} t\right)=0$, the four local equilibrium points of the symbiosis evolution of different symbiosis units in the social media ecological network can be obtained, namely, $E_{1}(0,0), E_{2}\left(N_{1}^{*}, 0\right), E_{3}\left(0, N_{2}^{*}\right)$, and $E_{4}\left(\left(N_{1}^{*}\left(1-\delta_{21}\right) / 1-\delta_{21} \delta_{12}\right),\left(N_{2}^{*}\left(1-\delta_{12}\right) / 1-\delta_{12}\right)\right)$. For the SCE described by a system of differential equations to participate in the evolution of the subject, the stability of its equilibrium point can be obtained by analyzing the local stability of the Jacobian matrix. Taking the partial derivatives of $N_{1}$ and $N_{2}$ for the differential equations, in turn, the Jacobian matrix of the symbiosis evolution between scientists and social media organizations in the SCE can be obtained as equation (5):
The method of using the Jacobian matrix to judge whether the equilibrium point is locally asymptotically stable is as follows: when the system equilibrium point makes $\operatorname{det}(J)>0$ and $\operatorname{tr}(J)<0$, then it is a stable equilibrium point. At this time, the equilibrium point is in a locally progressive and stable state. Therefore, in the SCE, the conditions for the symbiosis evolution between the group of scientists and social media organizations are $\operatorname{det}(J)>0$ and $\operatorname{tr}(J)<0$. The specific analysis results are shown in Table 2.

\section{Results and Discussion}

4.1. Simulation Analysis. The two types of symbiotic units, scientists and social media platforms, adopt a division of labor and cooperation to create new value, generate newly available resources, promote the evolution of symbiosis relationships, expand the scale of the SCE through the reuse of resources, and improve the operational efficiency of the SCE. This study uses MATLAB2019a to simulate the 
TABLE 1: The value range of the symbiosis coefficient and its corresponding symbiosis pattern. $\delta_{21}$ represents the influence coefficient of the scale change of population 2 on the growth of population 1 .

\begin{tabular}{|c|c|c|}
\hline Value range & $\begin{array}{l}\text { Symbiosis } \\
\text { pattern }\end{array}$ & Feature \\
\hline$\delta_{12}=0, \delta_{21}=0$ & Not symbiosis & Two symbiotic units developed independently \\
\hline$\delta_{12}>0, \delta_{21}>0$ & Not symbiosis & Two symbiotic units compete maliciously \\
\hline $\begin{array}{l}\delta_{12}<0, \delta_{21}>0 \text { or } \\
\delta_{12}>0, \delta_{21}<0\end{array}$ & Parasitic & Two types of symbiotic units, one reduced $(\delta>0)$ and the other benefited $(\delta<0)$ \\
\hline $\begin{array}{l}\delta_{12}<0, \delta_{21}=0 \text { or } \\
\delta_{12}=0, \delta_{21}<0\end{array}$ & Commensalism & $\begin{array}{l}\text { Two types of symbiotic units, one that benefits }(\delta<0) \text { and the other that does not affect } \\
\qquad(\delta=0)\end{array}$ \\
\hline$\delta_{12}<0, \delta_{21}<0$ & Mutualism & $\begin{array}{l}\text { Asymmetric mutualism between two types of symbiotic units }\left(\delta_{12} \neq \delta_{21}\right) \text {; symmetric } \\
\text { mutualism between two types of symbiotic units }\left(\delta_{12}=\delta_{21}\right)\end{array}$ \\
\hline
\end{tabular}

TABLE 2: Equilibrium and stability condition of SCE.

\begin{tabular}{|c|c|c|c|}
\hline Equilibrium & $\operatorname{det}(J)$ & $\operatorname{tr}(J)$ & $\begin{array}{l}\text { Stability } \\
\text { condition }\end{array}$ \\
\hline$E_{1}(0,0)$ & $\alpha_{1} \alpha_{2}$ & $\alpha_{1}+\alpha_{2}$ & Unstable \\
\hline$E_{2}\left(N_{1}^{*}, 0\right)$ & $-\alpha_{1} \alpha_{2}\left(1-\delta_{12}\right)$ & $-\alpha_{1}+\alpha_{2}\left(1-\delta_{12}\right)$ & $\delta_{12}>1$ \\
\hline$E_{3}\left(0, N_{2}^{*}\right)$ & $-\alpha_{1} \alpha_{2}\left(1-\delta_{21}\right)$ & $-\alpha_{2}+\alpha_{1}\left(1-\delta_{21}\right)$ & $\delta_{21}>1$ \\
\hline $\begin{array}{l}E_{4}\left(\left(N_{1}^{*}\left(1-\delta_{21}\right) / 1-\delta_{21} \delta_{12}\right)\right. \\
\left.\left(N_{2}^{*}\left(1-\delta_{12}\right) / 1-\delta_{12}\right)\right)\end{array}$ & $\left(\alpha_{1} \alpha_{2}\left(\delta_{21}-1\right)\left(\delta_{12}-1\right) / 1-\delta_{21} \delta_{12}\right)$ & $\left(\alpha_{1}\left(\delta_{21}-1\right)+\alpha_{2}\left(\delta_{12}-1\right) / 1-\delta_{21} \delta_{12}\right)$ & $\delta_{21}<1, \delta_{12}<1$ \\
\hline
\end{tabular}

differential equations (3) to explore how the community of scientists and social media platforms interact and evolve symbiosis under different values of $\delta_{12}$ and $\delta_{21}$ as shown in

$$
\begin{aligned}
& {\left[\begin{array}{l}
\frac{\mathrm{d} N_{1}(t)}{\mathrm{d} t} \\
\frac{\mathrm{d} N_{2}(t)}{\mathrm{d} t}
\end{array}\right]=\left[\begin{array}{l}
\alpha_{1} N_{1}(t)\left(1-\frac{N_{1}(t)}{N_{1}^{*}}-\delta_{21} \frac{N_{2}(t)}{N_{2}^{*}}\right) \\
\alpha_{2} N_{2}(t)\left(1-\frac{N_{2}(t)}{N_{2}^{*}}-\delta_{12} \frac{N_{1}(t)}{N_{1}^{*}}\right)
\end{array}\right],} \\
& {\left[\begin{array}{l}
N_{10} \\
N_{20}
\end{array}\right]=\left[\begin{array}{l}
100 \\
100
\end{array}\right] .}
\end{aligned}
$$

This paper uses the fourth-fifth-order Runge-Kutta algorithm to solve the numerical problem. It is assumed that the natural growth rates of the population of scientists participating in social media and the social media platforms are $\alpha_{1}=0.02$ and $\alpha_{2}=0.01$, respectively, the maximum growth scale of the two types of symbiosis units is $N_{1}^{*}=N_{2}^{*}=100$, when resources and environment allow, the iteration period is set to 1000 , and the simulation results are shown in Figures 3-8.

(1) Independent coexistence. Figure 3 shows the simulation results of the two types of symbiotic units in the SCE under the independent coexistence pattern. At this time, there is no symbiosis relationship between them, and the symbiosis coefficient between the scientist and the social media platform is 0 . In this pattern, the growth rate of the two types of symbiotic units does not affect each other, and the growing scale is only affected by its growth rate. In the independent coexistence pattern, the stable equilibrium point is $\left(N_{1}^{*}, N_{2}^{*}\right)$; that is, when the two

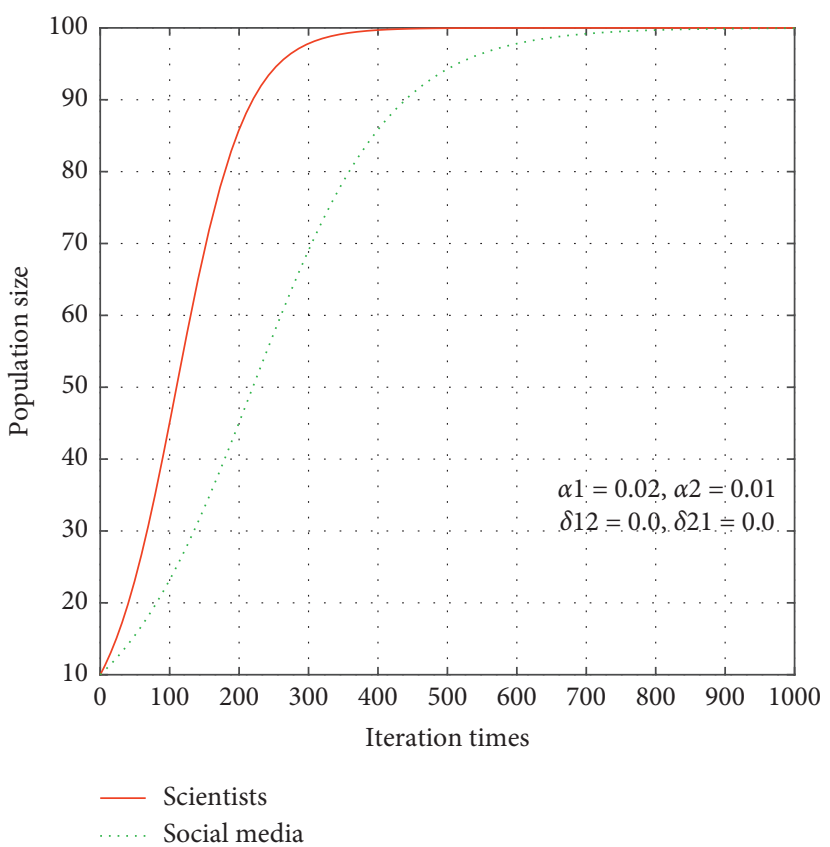

FIgURE 3: Independent coexistence pattern. Changes in the size of the two populations after 1000 iterations $\left(\alpha_{1}=0.02, \alpha_{2}=0.01\right.$, $\delta_{12}=0$, and $\left.\delta_{21}=0\right)$.

types of symbiotic units are in equilibrium, their growth scale reaches the upper limit. This pattern generally exists in the early stage of the formation of the SCE. Science communication activities not only are related to startups but also involve various entities in the system. Therefore, this pattern is very rare, short-lived, and unstable. 


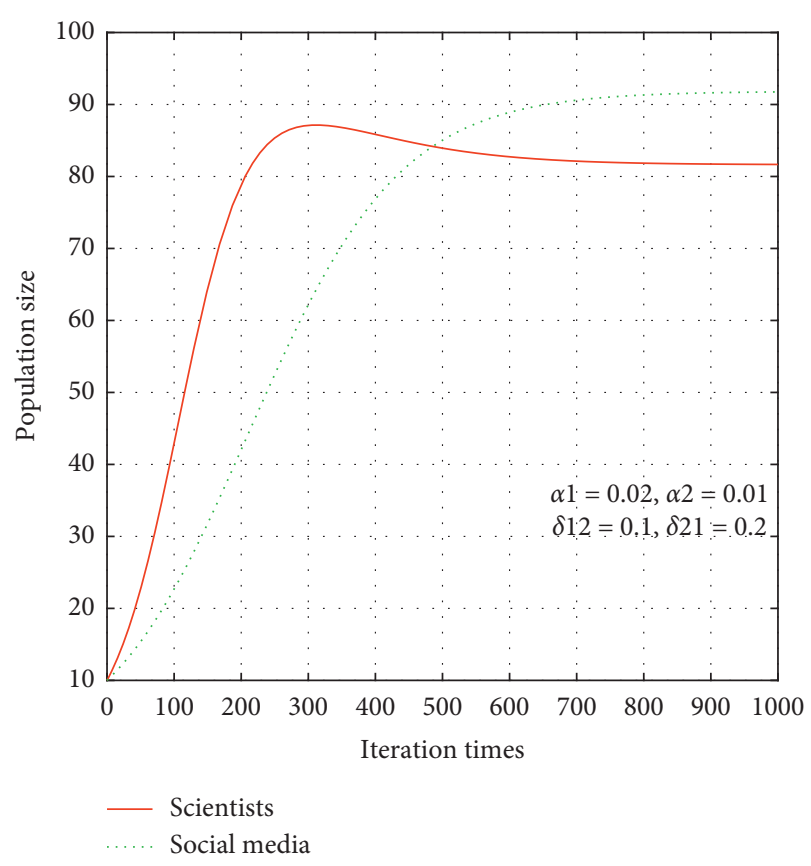

FIgure 4: Competitive coexistence pattern. Changes in the size of the two populations after 1000 iterations $\left(\alpha_{1}=0.02, \alpha_{2}=0.01\right.$, $\delta_{12}=0.1$, and $\delta_{21}=0.2$ ).

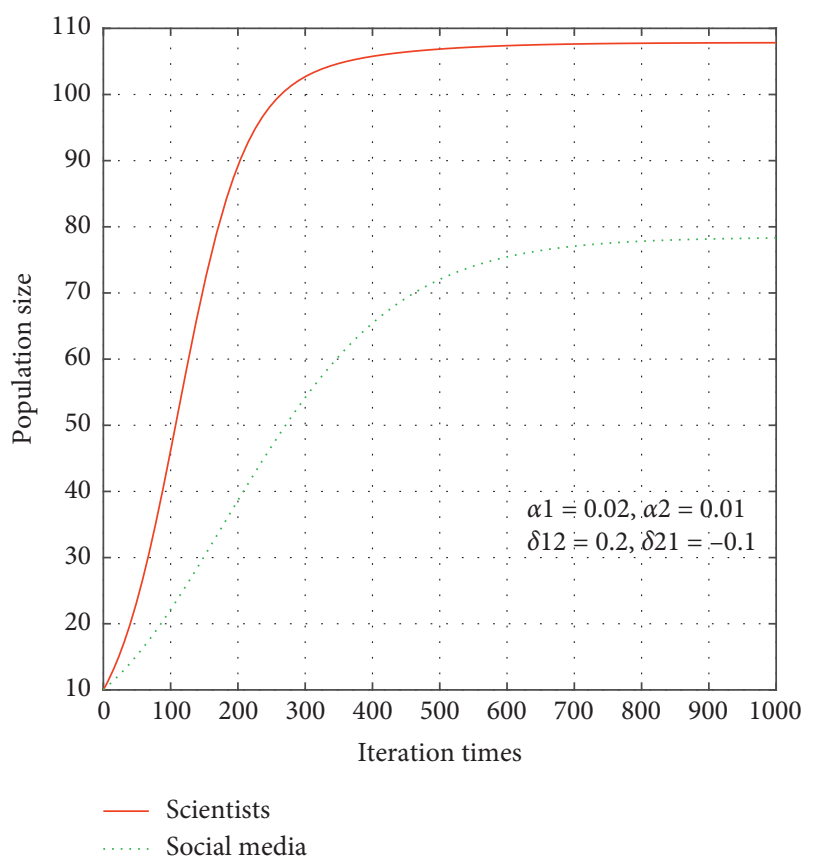

Figure 5: Parasitic coexistence pattern. Changes in the size of the two populations after 1000 iterations $\left(\alpha_{1}=0.02\right.$, $\alpha_{2}=0.01 \delta_{12}=0.2$, and $\delta_{21}=-0.1$ ).

(2) Competitive coexistence. In the evolution of the SCE, another pattern that does not exist in symbiosis is competition and coexistence. As shown in Figure 4, the symbiosis coefficient between the scientist group and the social media platform at this time is greater than 0 , the symbiosis coefficient of the participating

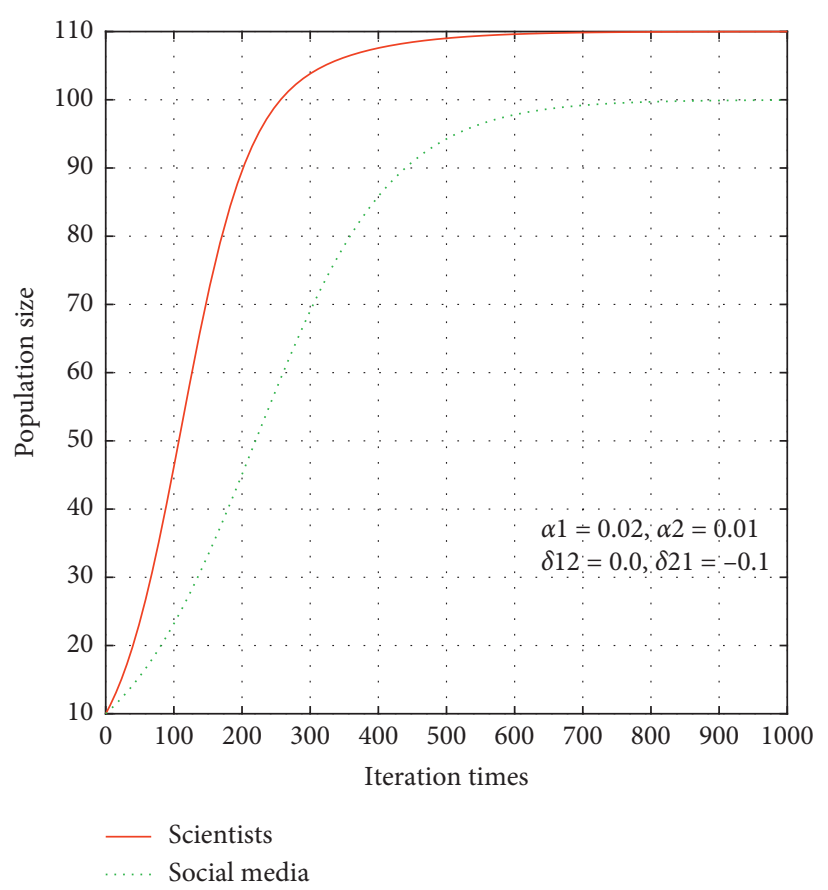

Figure 6: Commensalism pattern. Changes in the size of the two populations after 1000 iterations $\left(\alpha_{1}=0.02, \alpha_{2}=0.01, \delta_{12}=0\right.$, and $\delta_{21}=-0.1$ ).

social media scientists on the social media platform is greater than 1 , and the stable equilibrium point is $\left(N_{1}^{*}, 0\right)$. This pattern may exist when scientists have better resources and technology from other channels. Social media platforms tend to decline due to the consumption of a large number of resources, and the group of scientists has survived. However, due to mutual competition, the group of scientists has also suffered losses, and it is difficult to exceed the maximum scale under its constraints $N_{1}^{*}$.

(3) Parasitic coexistence. Figure 5 shows the simulation results of the parasitic symbiosis pattern of two types of symbiotic units in the SCE. In this pattern, the social media platforms have a positive symbiosis effect on the group of scientists participating in it, while the group of scientists cannot give equal returns to the social media platforms and cause a negative symbiosis effect on it. $0<\delta_{12}<1$ and $\delta_{21}<0$; the stable equilibrium point at this time is $\left(\left(N_{1}^{*}\left(1-\delta_{21}\right) / 1-\delta_{21} \delta_{12}\right),\left(N_{2}^{*}\left(1-\delta_{12}\right) / 1-\delta_{12}\right)\right)$. It can be seen that the upper limit of the growth scale of the scientist population under equilibrium conditions exceeds its maximum growth scale $N_{1}^{*}$. The social media platform has declined due to the consumption of the resources of the scientists participating in social media, and the growth scale is far below the maximum scale $N_{2}^{*}$. At the same time, with the advancement of science communication activities, the scale of the group of scientists participating in social media has gradually expanded. Although the parasitic behavior of the group of scientists has 


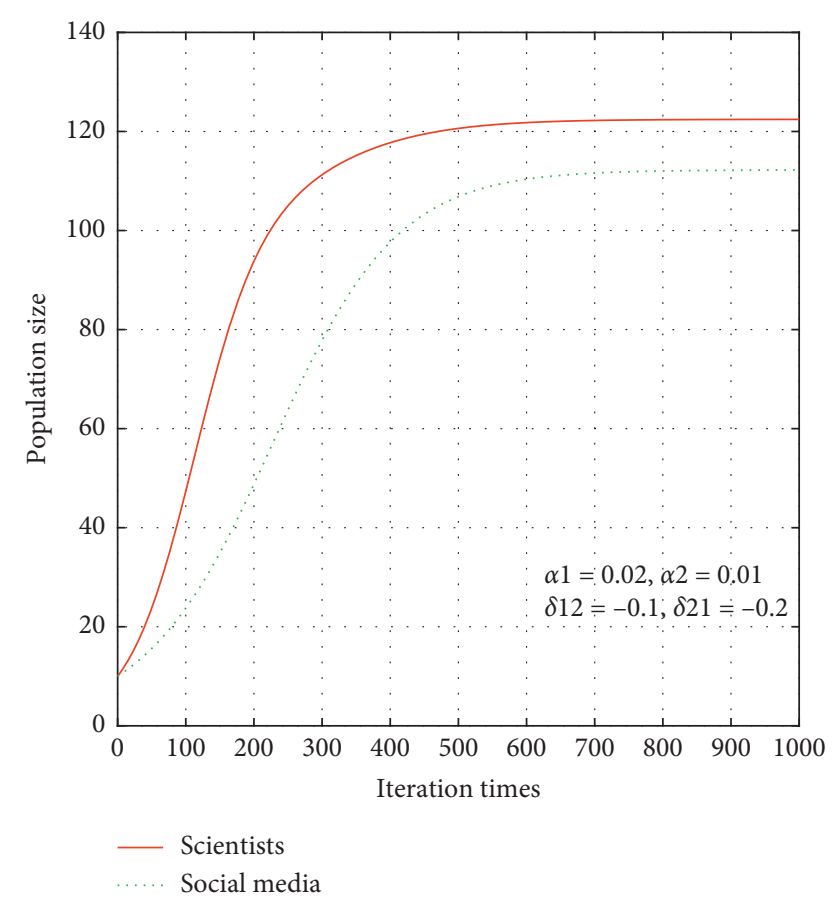

Figure 7: Asymmetric mutualism pattern. Changes in the size of the two populations after 1000 iterations $\left(\alpha_{1}=0.02, \alpha_{2}=0.01\right.$, $\delta_{12}=-0.1$, and $\delta_{21}=-0.2$ ).

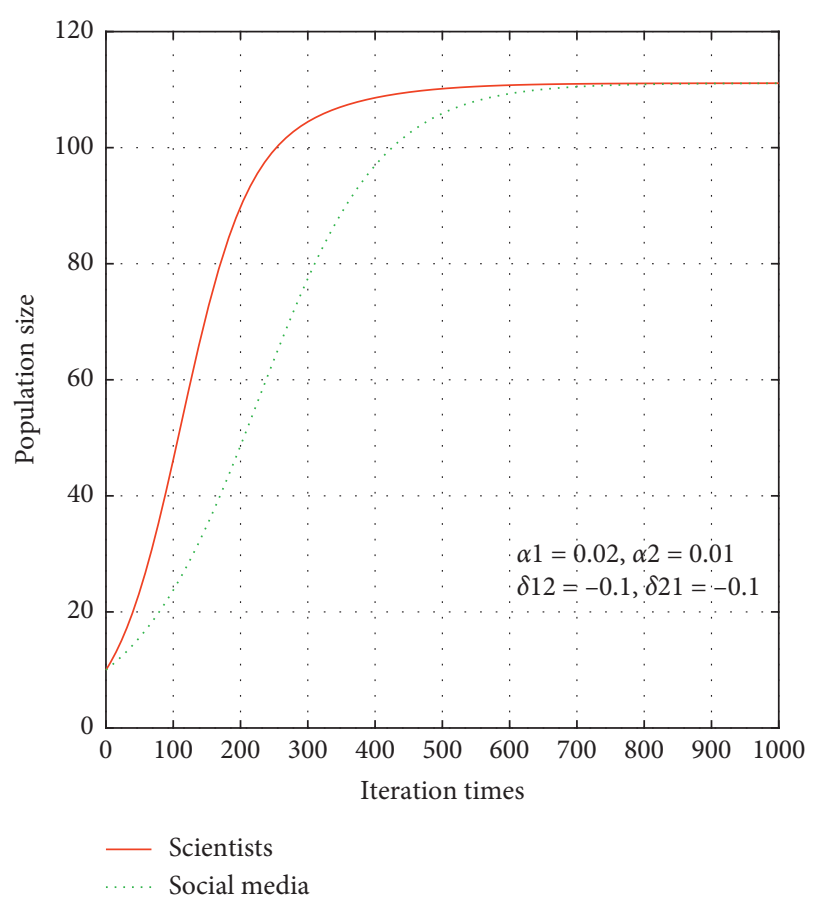

Figure 8: Symmetric mutualism pattern. Changes in the size of the two populations after 1000 iterations $\left(\alpha_{1}=0.02, \alpha_{2}=0.01\right.$, $\delta_{12}=-0.1$, and $\left.\delta_{21}=-0.1\right)$.

hindered the development of social media platforms, it has not formed a fatal threat, and the growth of social media platforms has been stable at a certain value below its maximum growth scale. At this time, the benefits of " $1+1>2$ " have not been realized between symbiotic units, and both of them need more resource interaction and value sharing to promote symbiosis evolution.

(4) Commensalism. As shown in Figure 6, in this pattern, one of the symbiosis coefficients between the social media platform and the participating scientists is less than zero, and the other is equal to zero. The stable equilibrium point at this time is $\left(N_{1}^{*}\left(1-\delta_{21}\right), N_{2}^{*}\right)$. The benefits brought by the symbiosis relationship made up for the damage that the social media platform suffered in the parasitic symbiosis pattern, and its growth scale returned to the maximum quality parameter $N_{2}^{*}$ of independent growth. The group of scientists participating in social media continues to benefit through symbiosis relationships, beyond the maximum growth scale $N_{1}^{*}$ when growing up independently. Under the commensalism pattern, the group of scientists continued to obtain technical, capital, and equipment support from the social media platform and gradually returned profits, talents, services, and content to compensate for the prerequisite investment of the social media platforms. The two types of symbiotic units get through the most difficult period for science communication and transit into the growth phase.

(5) Asymmetric mutualism. Figure 7 shows the simulation results of the asymmetric mutualism between scientists participating in social media and social media platforms. The symbiosis coefficients of the two types of symbiosis units are both less than 0 , but not equal. The stable equilibrium point at this time is $\left(\left(N_{1}^{*}\left(1-\delta_{21}\right) / 1-\delta_{21} \delta_{12}\right),\left(N_{2}^{*}\left(1-\delta_{12}\right) / 1-\delta_{12}\right)\right)$. The growth scale of the two types of symbiotic units exceeds the maximum scale of independent development under the constraints of their respective resources and environments. The upper limit of the size of each symbiotic unit is related to the symbiosis coefficient. The greater the absolute value of the symbiosis coefficient, the higher the upper limit of its growth scale. At the same time, with the withdrawal of funds and technologies from social media platforms, there are more options to choose from, and the growth momentum is accelerating. At this time, the evolution of the SCE has gradually entered a mature stage, and the symbiosis relationship between scientists participating in social media and social media platforms has begun to enter a win-win pattern.

(6) Symmetric mutualism. Symmetric mutualism is the highest level pursued in the process of symbiosis evolution. As shown in Figure 8, the symbiosis coefficients of the two types of symbiotic units are both less than zero and equal. The growth scale of the two types of symbiotic units not only exceeds the maximum quality parameter of independent development but also tends to be consistent. In the mutualism pattern, scientists involved in social 
media have close cooperation, mutual promotion, and mutual dependence on social media platforms. They have a good coordination mechanism and a resource sharing mechanism and ultimately achieve " $1+1>2$ " economic benefits.

From the above analysis, it can be concluded that the SCE based on social media is a complex system formed by the symbiosis and evolution of scientists and social media platforms. The coefficient of symbiosis between scientists participating in social media and social media platforms determines the result of the evolution of the system; different symbiosis relationships will eventually lead to different evolution directions and eventually reach an equilibrium state. The two types of symbiosis units under the mutually beneficial symbiosis pattern have the largest growth rate, which is the best direction for the evolution of social media ecological symbiosis.

\section{Conclusions}

This study summarized the concept of the SCE based on the actual situation, discussed the symbiosis evolution relationship between scientists and social media platforms in the SCE based on the Lotka-Volterra model within the theoretical framework of symbiosis theory, and calculated the equilibrium point and stability conditions of the symbiosis evolution with the Jacobian matrix. Comparing the symbiosis evolution pattern under different coefficients, we got some important conclusions as follows.

(1) The SCE in the context of science communication is composed of symbiotic units including scientists and social media platforms and interacts through different symbiosis patterns under the influence of economic, policy, and cultural symbiosis environments. Eventually, a self-organizing, adaptive coordination system is formed. The process of system integration is bound to go through stages of formation, growth, and maturity. The symbiosis relationship between the group of scientists and social media platforms will directly affect the development of the SCE. (2) The symbiosis coefficient between scientists and social media determines the equilibrium result of the symbiosis and evolution of the SCE. Independent coexistence, competitive coexistence, parasitic coexistence, and commensalism cannot promote the virtuous circle of the SCE, only if under the mutualism pattern, scientists and social media platforms promote each other's population size, which can achieve sustainable science communication. (3) The SCE based on social media can only produce economic benefits of " $1+1>2$ " under the symmetric mutualism pattern. The growth of scientists and social media organizations under the symmetric mutualism pattern is more stable and abundant than that of other patterns.

Generally, subsequent research can be carried out from the following aspects: (1) Introduce the research results of this study into specific practical situations for analysis. For example, we could conduct an empirical analysis of the number of scientists participating in social media for scientific communication and the number of social media platforms during a period, to make a useful supplement to the simulation results. (2) Explore the evolution of the SCE under the symbiosis organization mode, and discuss how it evolves from point symbiosis, intermittent symbiosis, and continuous symbiosis to integrated symbiosis. (3) Refer to the existing research on complex systems, explore the competition and cooperation mechanism and metabolism mechanism in the SCE, and provide corresponding suggestions for more effectively promoting the virtuous circle of science communication.

\section{Data Availability}

The data used to support the findings of this study are included within the article.

\section{Conflicts of Interest}

The authors declare that there are no conflicts of interest regarding the publication of this paper.

\section{Acknowledgments}

This study was supported by the National Natural Science Foundation of China (no. 71972148).

\section{References}

[1] F. El Kihal, I. Abouelkheir, M. Rachik, and I. Elmouki, "Role of media and effects of infodemics and escapes in the spatial spread of epidemics: a stochastic multi-region model with optimal control approach," Mathematics, vol. 7, 2019.

[2] A. Orben, "Teenagers, screens and social media: a narrative review of reviews and key studies," Social Psychiatry and Psychiatric Epidemiology, vol. 55, no. 4, pp. 407-414, 2020.

[3] J. Maier and B. Rittberger, "Shifting europe's boundaries," European Union Politics, vol. 9, no. 2, pp. 243-267, 2008.

[4] C. Puschmann, "Analyzing political communication with digital trace data: the role of twitter messages in social science research," Information, Communication \& Society, vol. 19, no. 12, pp. 1691-1692, 2015.

[5] D. Sgroi, "Social network theory, broadband and the future of the World Wide Web," Telecommunications Policy, vol. 32, no. 1, pp. 62-84, 2008.

[6] A. I. Cristea, D. Katsaros, and Y. Manolopoulos, "Introduction to the special issue of the world wide web journal on "social media preservation and applications"," World Wide Web, vol. 17, no. 4, pp. 691-693, 2014.

[7] C. Leppanen, D. M. Frank, J. J. Lockyer et al., "Media representation of hemlock woolly adelgid management risks: a case study of science communication and invasive species control," Biological Invasions, vol. 21, no. 2, pp. 615-624, 2018.

[8] P. Hunter, "The growth of social media in science," EMBO Reports, p. 21, 2020.

[9] V. Cheplygina, F. Hermans, C. Albers, N. Bielczyk, and I. Smeets, "Ten simple rules for getting started on Twitter as a scientist," Plos Comput Biol, vol. 16, Article ID e1007513, 2020.

[10] C. R. Sugimoto, S. Work, V. Larivière, and S. Haustein, "Scholarly use of social media and altmetrics: a review of the literature," Journal of the Association for Information Science and Technology, vol. 68, no. 9, pp. 2037-2062, 2017. 
[11] X. Lu, C. W. Phang, and J. Yu, "Encouraging participation in virtual communities through usability and sociability development," ACM SIGMIS Database: The DATABASE for Advances in Information Systems, vol. 42, no. 3, pp. 96-114, 2011.

[12] S. Choi, H. Lee, and Y. Yoo, "The impact of information technology and transactive memory systems on knowledge sharing, application, and team performance: a field study," Mis Quarterly, vol. 34, no. 4, pp. 855-870, 2010.

[13] A. Gruzd, K. Staves, and A. Wilk, "Connected scholars: examining the role of social media in research practices of faculty using the UTAUT model," Computers in Human Behavior, vol. 28, no. 6, pp. 2340-2350, 2012.

[14] R. B. Lee, R. Baring, M. S. Maria, and S. Reysen, "Attitude towards technology, social media usage and grade-point average as predictors of global citizenship identification in Filipino University Students," International Journal of Psychology, vol. 52, no. 3, pp. 213-219, 2017.

[15] C. Tenopir, R. Volentine, and D. W. King, "Social media and scholarly reading," Online Information Review, vol. 37, no. 2, pp. 193-216, 2013.

[16] E. C. M. Parsons, D. S. Shiffman, E. S. Darling, N. Spillman, and A. J. Wright, "How Twitter literacy can benefit conservation scientists," Conservation Biology, vol. 28, no. 2, pp. 299-301, 2014.

[17] K. Collins, D. Shiffman, and J. Rock, "How are scientists using social media in the workplace?" PLoS One, vol. 11, Article ID e0162680, 2016.

[18] J. Juenger and B. Faehnrich, "Does really no one care? analyzing the public engagement of communication scientists on Twitter," New Media \& Society, vol. 22, pp. 387-408, 2019.

[19] B. Faehnrich and C. Luthje, "Roles of social scientists in crisis media reporting-the case of the German populist radical right movement PEGIDA," Science Communication, vol. 39, pp. 415-442, 2017.

[20] X. Liang, L. Y.-F. Su, S. K. Yeo et al., "Building buzz," Journalism \& Mass Communication Quarterly, vol. 91, no. 4, pp. 772-791, 2014.

[21] J. M. Heemstra, "A scientist's guide to social media," ACS Central Science, vol. 6, no. 1, pp. 1-5, 2020.

[22] I. Rowlands, D. Nicholas, B. Russell, N. Canty, and A. Watkinson, "Social media use in the research workflow," Learned Publishing, vol. 24, no. 3, pp. 183-195, 2011.

[23] B. Beck-Winchatz, "Involvement of scientists in the NASA Office of Space Science education and public outreach program," Nuclear Physics B - Proceedings Supplements, vol. 138, pp. 451-453, 2005.

[24] C. Stylinski and A. Johnson, "Impacts of a comprehensive public engagement training and support program," International Journal of Science Education, vol. 4, no. 8, pp. 340-354, 2018.

[25] A. T. Taylor and S. M. Sammons, "Bridging the gap between scientists and anglers: the black bass conservation committee's social media outreach efforts," Fisheries, vol. 44, no. 1, pp. 37-41, 2019.

[26] J. T. H. Wang, C. J. Power, C. M. Kahler et al., "Communication ambassadors-an Australian social media initiative to develop communication skills in early career scientists," Journal of Microbiology \& Biology Education, vol. 19, 2018.

[27] B. Smith, N. Baron, C. English et al., "COMPASS: navigating the rules of scientific engagement," PLoS Biology, vol. 11, Article ID e1001552, 2013.

[28] K. D. Heath, E. Bagley, A. J. M. Berkey et al., "Amplify the signal: graduate training in broader impacts of scientific research," BioScience, vol. 64, no. 6, pp. 517-523, 2014.
[29] A. Baram-Tsabari and B. V. Lewenstein, "Science communication training: what are we trying to teach?" International Journal of Science Education, Part B, vol. 7, no. 3, pp. 285-300, 2017.

[30] C. Stylinski, M. Storksdieck, N. Canzoneri, E. Klein, and A. Johnson, "Impacts of a comprehensive public engagement training and support program on scientists' outreach attitudes and practices," International Journal of Science Education, Part B, vol. 8, no. 4, pp. 340-354, 2018.

[31] E. Salas, S. I. Tannenbaum, K. Kraiger, and K. A. SmithJentsch, "The science of training and development in organizations," Psychological Science in the Public Interest, vol. 13, no. 2, pp. 74-101, 2012.

[32] J. Wan, Y. Lu, B. Wang, and L. Zhao, "How attachment influences users' willingness to donate to content creators in social media: a socio-technical systems perspective," Information \& Management, vol. 54, no. 7, pp. 837-850, 2017.

[33] A. Susarla, J.-H. Oh, and Y. Tan, "Social networks and the diffusion of user-generated content: evidence from YouTube," Information Systems Research, vol. 23, no. 1, pp. 23-41, 2012.

[34] S. Krishnamurthy and A. K. Tripathi, "Monetary donations to an open source software platform," Research Policy, vol. 38, no. 2, pp. 404-414, 2009.

[35] Q. K. Mahmood, S. R. Jafree, and M. M. Sohail, "Pakistani youth and social media addiction: the validation of bergen Facebook addiction scale (BFAS)," International Journal of Mental Health and Addiction, 2020.

[36] M. Kaakinen, A. Sirola, I. Savolainen, and A. Oksanen, "Shared identity and shared information in social media: development and validation of the identity bubble reinforcement scale," Media Psychology, vol. 23, no. 1, pp. 25-51, 2018.

[37] B. B. Dedeoğlu, B. Taheri, F. Okumus, and M. Gannon, "Understanding the importance that consumers attach to social media sharing (ISMS): scale development and validation," Tourism Management, p. 76, 2020.

[38] L. J. GOFF, "Symbiosis and parasitism: another viewpoint," BioScience, vol. 32, no. 4, pp. 255-256, 1982.

[39] D. Leidner, "Review and theory symbiosis: an introspective retrospective," Journal of the Association for Information Systems, vol. 19, no. 06, pp. 552-567, 2018.

[40] L. Vančurová, V. Kalníková, O. Peksa et al., "Symbiosis between river and dry lands: phycobiont dynamics on river gravel bars," Algal Research, p. 51, 2020.

[41] P. Kerdlap, J. S. C. Low, and S. Ramakrishna, "Life cycle environmental and economic assessment of industrial symbiosis networks: a review of the past decade of models and computational methods through a multi-level analysis lens," The International Journal of Life Cycle Assessment, vol. 25, no. 9, pp. 1660-1679, 2020.

[42] J. Zhang and W. L. Chen, "The study on integration of supply chain based on the symbiosis theory," Applied Mechanics and Materials, vol. 275-277, pp. 2706-2709, 2013.

[43] G. Fontaine, M.-A. Maheu-Cadotte, A. Lavallée et al., "Communicating science in the digital and social media ecosystem: scoping review and typology of strategies used by health scientists," JMIR Public Health and Surveillance, vol. 5, no. 3, p. e14447, 2019.

[44] K. M. Meyer, A. M. Hopple, A. M. Klein, A. H. Morris, S. D. Bridgham, and B. J. M. Bohannan, "Community structure - ecosystem function relationships in the Congo Basin methane cycle depend on the physiological scale of function," Molecular Ecology, vol. 29, no. 10, pp. 1806-1819, 2020 . 
[45] M. Loreau, "Coexistence of multiple food chains in a heterogeneous environment: interactions among community structure, ecosystem functioning, and nutrient dynamics," Mathematical Biosciences, vol. 134, no. 2, pp. 153-188, 1996.

[46] X. Tan, W. Qin, G. Tang, C. Xiang, and X. Liu, "Models to assess the effects of nonsmooth control and stochastic perturbation on pest control: a pest-natural-enemy ecosystem," Complexity, vol. 2019, 14 pages, 2019.

[47] P. Wang, W. Qin, and G. Tang, "Modelling and analysis of a host-parasitoid impulsive ecosystem under resource limitation," Complexity, vol. 2019, 12 pages, 2019.

[48] J.-L. Usó-Doménech, J.-A. Nescolarde-Selva, M. Lloret-Climent, H. Gash, and K. Alonso-Stenberg, "Indirect effects, biotic inferential interactions and time functions in $\mathrm{H}$-semiotic systems: ecosystems case," Mathematics, vol. 7, 2019.

[49] A. Voroshilova and J. Wafubwa, "Discrete competitive lotka-volterra model with controllable phase volume," Systems, vol. 8, 2020.

[50] S. Guo and S. Yan, "Hopf bifurcation in a diffusive LotkaVolterra type system with nonlocal delay effect," Journal of Differential Equations, vol. 260, no. 1, pp. 781-817, 2016.

[51] G. Bernardo and S. D'Alessandro, "Systems-dynamic analysis of employment and inequality impacts of low-carbon investments," Environmental Innovation and Societal Transitions, vol. 21, pp. 123-144, 2016.

[52] N. T. Khan, Y. H. Kim, and Y. B. Kim, "The dynamic impact of low-cost carriers on full-service carriers and the tourism industry of South Korea: a competitive analysis using the Lotka-Volterra model," Asia Pacific Journal of Tourism Research, vol. 23, no. 7, pp. 656-666, 2018.

[53] V. Assumma, M. Bottero, G. Datola, E. De Angelis, and R. Monaco, "Dynamic models for exploring the resilience in territorial scenarios," Sustainability, vol. 12, 2019.

[54] A. Marasco and A. Romano, "Deterministic modeling in scenario forecasting: estimating the effects of two public policies on intergenerational conflict," Quality \& Quantity, vol. 52, no. 5, pp. 2345-2371, 2017.

[55] J. Ditzen, "Cross-country convergence in a general LotkaVolterra model," Spatial Economic Analysis, vol. 13, no. 2, pp. 191-211, 2017.

[56] N. Christodoulakis, "Conflict dynamics and costs in the Greek Civil war 1946-1949," Defence and Peace Economics, vol. 27, no. 5, pp. 688-717, 2015.

[57] G. Faye and M. Holzer, "Asymptotic stability of the critical pulled front in a Lotka-Volterra competition model," Journal of Differential Equations, vol. 269, no. 9, pp. 6559-6601, 2020. 